\title{
EDITORIAL
}

\section{Stewards of a changing planet: commentaries from ISME13 Plenary Lecturers}

The ISME Journal (2010) 4, 1079-1080; doi:10.1038/ismej.2010.114

Microorganisms are increasingly recognized as key stewards of the biosphere. Their roles range from maintenance of human, animal and plant health to driving biogeochemical cycles at the global scale. They are also key responders to the changing climate in ways that may prove beneficial, such as increased $\mathrm{CO}_{2}$ fixation, or ultimately detrimental, such as shifts in the population and distribution of disease-causing microorganisms. With this in mind, The ISME13 Symposium (22-27 August 2010, Seattle, WA, USA) brought together the international community of microbial ecologists under the theme of microbial contributions to planetary health. ISME13 had an extensive international scientific program that included 30 invited sessions with speakers from around the globe and 3 interactive poster sessions from which the David C White Most Innovative Student poster was selected. The topics for these sessions ranged from disease ecology to climate change-induced feedbacks in microbial processes and also highlighted petroleum microbiology, including late-breaking results from the recent Deepwater Horizon oil spill in the Gulf of Mexico. These sessions were complimented with a series of roundtable discussions that explored topics including recent advances in fluorescence in situ hybridization, environmental metagenomics and proteomics, as well as diverse scientific issues, such as syntrophy and the evolution of extemophiles.

ISME13 also featured a series of plenary talks by leading scientists who provided inspiring lectures on exciting current topics in microbial ecology. These plenary lecturers were invited to contribute commentaries to The ISME Journal that reflected the primary conclusions and opinions conveyed in their lectures. The resulting commentaries, included in this issue of The ISME Journal, range from molecular ecological analyses of important biogeochemical processes to the structure and function of microbial communities in biofilms, soil and the animal gut to ecosystem level responses to climate change, as briefly described below.

Ian R Sanders from the University of Lausanne, Switzerland, discusses new knowledge about the natural variation and diversity of arbuscular mycorrhizal fungi. He focuses on recent advances that allow manipulation of this natural variation to 'design' specific arbuscular mycorrhizal fungi that have optimal growth-promoting effects in symbiosis with specific crop plants.

Big sulfur bacteria are the topic of discussion in the commentary provided by Bo Barker Jørgensen (Aarhus University, Denmark). These microbes are particularly interesting, not only because of their unusually large size but also because of their unique specialization to life in specific zones that contain the nutrients they require for survival. Their functional roles and survival mechanisms are discussed in an evolutionary context. In recognition of his outstanding lifetime contributions to microbial ecology, Professor Jørgensen was awarded the Jim Tiedje award, which was presented at the ISME13 symposium.

Microbial communities in nature often reside in biofilms, with a complex interplay between cells in the biofilm. This topic is addressed in a commentary provided by J William 'Bill' Costerton (AlleghenySinger Research Institute, Pittsburgh, PA, USA) and his colleagues. They focus on Myxococcus xyanthus biofilms as a model for understanding the social behavior of cells residing in biofilms and discuss the use of three-dimensional imaging to better understand the roles of specific structures, such as vesicles, in biofilms.

Warwick F Vincent (Laval University, QC, Canada) provides a commentary on the impact of climate change on the ecology of Arctic microbial communities and processes. He focuses on the specific impact of declining Arctic sea ice and the microbes that live in this habitat. He also discusses how other Arctic habitats, including permafrost, are predicted to react to increasing global temperatures and stresses and that more attention is required to understand the microbial responses to these changes.

Ove Hoegh-Guldberg (University of Queensland, Australia) takes the discussion of climate change impacts to the next level with a thought-provoking commentary on shifts in ocean function that may have already begun because of climate change. He stresses the importance of understanding how changes in ocean temperatures and chemistry impact key populations and microbial systems and calls for immediate action to mitigate problems associated with climate change.

Christa Schleper, from the University of Vienna, Austria, provides an update on recent findings about the relative abundance and contributions of bacteria and archaea to ammonia oxidation in 
different habitats. She discusses new knowledge gained through the application of various molecular and genomic approaches to tease out the relative contributions of these two divisions of life to this key step in the global cycling of nitrogen.

Recently, there has been increasing interest in the ecology and function of microorganisms inhabiting the human gut, due to the importance of this relationship for health and disease. Jeff Gordon and his colleagues at the Washington University School of Medicine (St Louis, MO, USA) discuss the importance of germ-free 'gnotobiotic' mouse models for gaining an understanding of the complex interplay between the gut microbiome and the host. His commentary also includes practical information about how to obtain germ-free mice for the study of a humanized microbiota, or the impact of specific microbes of interest in the gut.

Additional plenary lecturers at ISME13 included Penny Chisholm (Massachusetts Institute of Technology, Cambridge, MA, USA), Thomas Schmidt from the Michigan State University (East Lansing, MI, USA) and Dr Rita Colwell (University of Maryland and Johns Hopkins University in Baltimore, MD, USA). Penny Chisholm's research focuses on the ubiquitous marine phototroph, Prochlorococcus. This organism is the dominant primary producer in the oceans, the smallest known phototroph and the most abundant photosynthetic organism on the planet. Over the past 10 years, the Chisholm laboratory has been developing Prochlorococcus as a model system for cross-scale systems biology to understand the biology of this bacterium from the genome level to the global scale. Tom Schmidt and colleagues are relating emergent properties of microbial communities to their genetic and organismal composition. Using a suite of metagenomic methods, they are investigating microbial communities in soil and the associated fluxes of methane, nitrous oxide and carbon dioxide-all greenhouse gases that are influenced by land management practices. The results fit a model in which the magnitude and stability of a process is related to the number of different microbes that mediate a given process. Ultimately, their research seeks to identify principles that explain patterns of microbial distribution in nature, including tradeoffs between metabolic power and efficiency. Rita Colwell and her colleagues investigate the ecology of the bacterium that causes cholera, Vibrio cholerae, and have traced this organism across the globe, from plankton populations in rivers to estuaries in Bangladesh. They have also linked cholera epidemiology to sea temperatures and plankton blooms, tying together global climate and disease. Taking this holistic perspective on pathogenesis into account has enabled researchers to begin to predict disease pandemics.

In summary, this unique collection of plenary lectures from ISME13, and the associated commentaries in this issue of ISME Journal, describe examples of exciting trends in the field of microbial ecology and provide important guidance for future research.

JK Jansson ${ }^{1}$ and JK Fredrickson ${ }^{2}$ ${ }^{1}$ Ecology Department, Earth Sciences Division, Lawrence Berkeley National Laboratory, Berkeley, CA, USA and ${ }^{2}$ Biological Sciences Division, Fundamental Science Directorate, Pacific Northwest National Laboratory, Richland, WA, USA E-mail: jim.fredrickson@pnl.gov 